\title{
Construcción de Proyectos de Vida Alternativos (PVA) en Urabá, Colombia: papel del sistema educativo en contextos vulnerables
}

\author{
Construction of Alternative Life Projects (ALP) in Urabá, Colombia: the role of the \\ educational system in vulnerable contexts
}

\section{Karen García-Yepes}

Facultad de Ciencias Sociales y Económicas, Universidad Juan de Castellanos, Colombia.

Correo electrónico: garciayepesk@gmail.com

\section{RESUMEN}

Este artículo surge como resultado de una Tesis Doctoral realizada entre 2013 y 2016 sobre el papel de la escuela en la construcción de los proyectos de vida en contextos vulnerables. El principal resultado del análisis sugiere que en estos entornos se producen Proyectos de Vida Alternativos (PVA) donde la niñez y la adolescencia están condicionadas por las circunstancias sociales y se alternan con las responsabilidades de la vida adulta a través de diversas estrategias de autosostenimiento. Se concluye que el rol de la escuela consiste en reproducir estas dificultades sociales estructurales o garantizar una formación integral y diferenciadora que aporte significativamente al curso vital del joven.

Palabras clave: Proyecto de vida, contexto social, contexto educativo, comunidad vulnerable, condiciones de vida.

\section{ABSTRACT}

This article has been originated as a result of a Doctoral Dissertation developed between 2013 and 2016 focused on the role of the school in the construction of life projects in vulnerable contexts. The main result of the analysis suggests that Alternative Life Projects (ALP) in these environments are produced where childhood and adolescence are conditioned by social circumstances and are alternated with the responsibilities of adulthood through diverse self-supporting strategies. As a conclusion, it is alleged that the role of the school consists of reproducing these structural social difficulties or guaranteeing an integral and differentiating formation that contributes significantly to the minor's vital course.

Key words: Life Project, Social environment, Educational environment, disadvantaged groups, living conditions. 


\section{INTRODUCCIÓN}

El objetivo de este artículo consiste en identificar el papel de la escuela en la construcción de los proyectos de vida en contextos vulnerables. Se trata de un análisis pertinente, por cuanto el centro educativo es una organización social que no sólo es escenario de adquisición de conocimientos, sino que también es un entorno donde se desarrollan habilidades para la vida y la socialización. Por ello, este análisis es importante en la medida en que permite indagar el rol que cumple la escuela en la formación no solo de estudiantes, sino también de seres humanos y ciudadanos que conviven en comunidad. Con respecto a este punto, es de interés relacionar este estudio con los procesos de empoderamiento y liderazgo desde el entorno educativo como estrategia para incidir en las trayectorias vitales de los jóvenes.

Por ello, en este artículo se define conceptualmente el significado del Proyecto de vida a partir de tres dimensiones: valoración de sí mismo, proyección futura y relación con el contexto social y educativo. Posteriormente, se analiza cómo se evidencia este proceso entre los jóvenes de la Institución Educativa El Playón, municipio de Carepa, región de Urabá, departamento de Antioquia (Colombia). Esto es oportuno por cuanto se trata de un contexto donde estos planes vitales entran en tensión con un entorno social complejo donde aún se evidencian las repercusiones del conflicto armado y de la pobreza económica. De este modo, en el marco del posconflicto en Colombia, adquiere especial trascendencia preguntarse sobre la incidencia de la escuela en la construcción de los cursos vitales y en los procesos de desarrollo comunitario desde la base.

Así, se hace un análisis sobre los procesos de construcción de Proyectos de Vida Alternativos donde la niñez y la adolescencia entran en interacción con las responsabilidades de la vida adulta. En esa medida, se estudian tres factores: primero, limitaciones económicas y estrategias de trabajo informal, segundo, características del contexto social y de las relaciones interpersonales; y por último, impacto de la perspectiva de género en el entorno observado. Todo ello con el objetivo de identificar de qué manera el sistema educativo limita o favorece la construcción del proyecto de vida en contextos vulnerables.

\section{FUNDAMENTACIÓN TEÓRICA}

\subsection{DEFINICIONES Y TENSIONES EN LA CONSTRUCCIÓN DEL PROYECTO DE VIDA}

El proyecto de vida se puede concebir como un proceso que estructura tanto las expectativas vitales como las estrategias para lograr dichos objetivos en el marco de un contexto social. En ese sentido la construcción de un itinerario vital, no se puede comprender si no es con relación a las realidades de ese entorno. Por ello, este constructo personal está construido tanto por las aspiraciones como por las posibilidades de concretar estas proyecciones de vida. De este modo, no es posible pensar en la construcción de un proyecto de vida si no es con relación a las posibilidades concretas de realización de acuerdo con la realidad social e histórica a la que pertenece el individuo. Desde esta perspectiva, el proyecto de vida representa la relación del individuo consigo mismo y con la comunidad a la que pertenece, definiendo las posibilidades para lograr esas aspiraciones (D’Angelo, 2002). Así, es un constructo que adquiere sentido a partir de la relación del individuo con su entorno y con las posibilidades que éste ofrece. Es por esto que la configuración y la dirección de 
este Proyecto se vinculan con la situación social del individuo con relación al presente y al futuro que se pretende construir (D’Angelo, 2002). Interacción social e histórica que orienta los caminos de la experiencia individual.

Otro concepto que se relaciona con el de Proyecto de Vida es el de itinerario vital. Esta idea hacer referencia a un actor social como sujeto histórico que construye sus decisiones y acciones pero bajo determinaciones de orden familiar, social, estructural y simbólico (Casal, García, Merino y Quesada, 2006). Así, se produce una tensión entre lo proyectado y lo posible en medio de unos condicionamientos concretos. Se trata entonces de coyunturas vitales donde las realizaciones personales también dependen de un contexto que puede convertir las aspiraciones personales en posibles o irrealizables (JohnsonHanks, 2002). Diferencia esencial entre la visión generalizante del proyecto de vida donde la experiencia humana es concebida de manera lineal y la visión integral donde el individuo es comprendido en su dimensión social e histórica.

Un último concepto es el de curso de vida. Según Elder, el desarrollo personal es un proceso que se produce a lo largo de la vida, está ligado al tiempo y lugar que pertenece el individuo, se vincula con su red de relaciones y articula las decisiones o acciones con las posibilidades y restricciones de un contexto (1998). En esa medida, la realización personal también debe comprenderse en el marco de las oportunidades que ofrece el entorno. Por consiguiente, los cambios en la vida se producen en el largo plazo, se articulan con el ámbito familiar o social y combinan la acción individual con condicionamientos sociales, estructurales o institucionales (Mayer, 2009). Por ello, es inevitable redimensionar el potencial transformador sobre la propia realidad pero al mismo tiempo es pertinente reconocer las circunstancias limitantes o realizadoras en la construcción del curso vital. Así, la vida individual se articula con las estructuras sociales que enmarcan la participación de las personas como miembros de un contexto, permitiendo una mirada más compleja de la experiencia vital en vez de una posición generalizadora (Sepúlveda, 2010). Pues en efecto, las posibilidades de inclusión social también están determinadas por políticas económicas o educativas que no siempre responden a las realidades sociales e individuales.

\subsection{DIMENSIONES DEL PROYECTO DE VIDA}

De igual manera, la noción de Proyecto de Vida se entiende con relación a las estrategias, actividades, comportamientos y pautas que desarrolla un individuo con relación a los objetivos propuestos (Leiva, 2012). Es así que el individuo logra estructurar sus aspiraciones vitales en función de las posibilidades y acciones reales para concretar estos propósitos. En consecuencia, se promueve la posibilidad de tomar consciencia frente a las posibilidades de lograrlas desde el reconocimiento de las habilidades y dificultades que pueden intervenir en este proceso (Pardo, 1999). En ese sentido, se alude inevitablemente a la posibilidad de reconocer falencias y dificultades inevitables en la construcción de esa proyección vital. De esa forma, dicha construcción está vinculada a la interacción entre valores, aspiraciones y expectativas para determinar pautas de acción frente a las posibilidades y oportunidades de vida (D’Angelo, 2000). En efecto, desde el reconocimiento de esos aspectos por mejorar se puede aspirar a reconocer posibilidades y obstáculos reales de acuerdo con lo proyectado. El Proyecto Vital se constituye entonces como construcción del individuo sobre sus expectativas vitales, determinando sus valores esenciales y sus pautas de acción frente a estas proyecciones. 
Estudios Pedagógicos XLIII, Nº 3: 153-173, 2017

CONSTRUCCIÓN DE PROYECTOS DE VIDA ALTERNATIVOS (PVA) EN URABÁ, COLOMBIA: PAPEL DEL SISTEMA EDUCATIVO EN CONTEXTOS VULNERABLES

En este sentido, se pueden identificar dos componentes esenciales del Proyecto Vital. En primer lugar, es un direccionamiento de los valores esenciales y una construcción del plan de acción individual en función de las metas y las aspiraciones personales. En este sentido, no es algo estático y se va construyendo a lo largo de la vida de acuerdo con las experiencias que permiten obtener logros o replantear lo planeado (Lomelí-Parga, LópezPadilla y Valenzuela-González, 2016). Es decir, adquiere una redimensión continua a partir del devenir de la cotidianidad. Con respecto a esta idea, también cabría decir que además de desarrollarse en el transcurso del ciclo vital, también es un proceso no lineal que implica la búsqueda de estrategias para alcanzar objetivos y superar dificultades (Romero, 2004). En segundo lugar, es un constructo que depende de la situación social del individuo en su entorno, definiendo las posibilidades de realización de esta proyección. Así, se evidencia la interacción de las posibles vivencias, logros y posibilidades con el contexto social al que pertenece el individuo (D'Angelo, 2000; Romero, 2004).

Además, cabe resaltar que el proyecto de vida se construye sincrónicamente en múltiples escenarios vitales. Por ello adquiere un carácter transversal y multidimensional que abarca diferentes áreas y dimensiones de la vida (Lomelí-Parga et al. 2016). Es en el marco de la interacción entre éstas que se van configurando las diversas trayectorias de los individuos. De esta manera, la construcción de ese futuro vital puede abarcar la esfera sentimental, la sociopolítica, la cultura y la profesional (D'Angelo, 2000; Leiva, 2012). Se produce entonces una interrelación de factores que pueden generar tensiones entre sî o pueden llegar a ser simultáneos en un mismo momento vital. De igual modo, además de las mencionadas, se puede tener en cuenta la espiritual, la intelectual, la familiar, y la económica (Castañeda, 2001). Por tanto, a partir de una relación interdependiente donde intervienen realidades concretas, aspiraciones y estrategias, el individuo propone alternativas para hacer frente a los obstáculos y los retos del diario vivir.

Al tener en cuenta que la construcción del proyecto de vida se compone por dimensiones familiares, sociales, económicas y profesionales, es inevitable desligar este análisis de la perspectiva de género. En efecto, este enfoque surge como factor de análisis pues durante generaciones se han producido avances en cuento a la igualdad de oportunidades laborales y formativas entre hombres y mujeres. No obstante, también es claro que aún queda eliminar algunas barreras en diversos órdenes de la vida para alcanzar la plena igualdad en diversos ámbitos (Santana, 2002). Así, es imprescindible buscar las condiciones para favorecer el acceso a la educación, a la participación política y al mercado laboral independientemente del sexo. En consecuencia, el reto desde los escenarios educativos consiste en coordinar acciones orientadas a favorecer el acceso igualitario de hombres y mujeres a diversas esferas de la vida personal y profesional en condiciones de justicia y equidad (Santana, Feliciano y Santana, 2012). Precisamente, desde la construcción real de posibilidades de inclusión social, se puede garantizar el acceso de ellas a escenarios de los que han sido tradicionalmente excluidas.

Además de la perspectiva de género, es inevitable desligar este análisis de su relación con la edad y los condicionamientos sociales. De acuerdo con D'Angelo, es precisamente en la juventud donde la construcción del proyecto de vida tiene especial importancia pues está en estado de elaboración casi total (1986). En su paso de la niñez a la adolescencia y la juventud, se presenta ante el joven un abanico de posibilidades y de dificultades que se manifiesta como reflejo de aspiración o de temor. Por ello, es la etapa de la vida donde la acción presente se enfoca más hacia el futuro, pues implica una proyección 
hacia la trayectoria vital en proceso de construcción (D’Angelo, 1986). La posibilidad de consecución o de frustración se visualiza a través de una tensión entre lo que aspira a ser y las oportunidades reales que ofrece el entorno.

Por consiguiente, es en esta etapa de la vida donde esa planeación futura adquiere mayor complejidad pues entra en juego una contradicción constante entre la participación o la exclusión social según sus posibilidades de acceder al mercado laboral (Leiva, 2012). Así, teniendo en cuenta las condiciones reales de formación educativa o de acceso al empleo, se pueden definir las características de ese proyecto vital. En consecuencia, se evidencia que la construcción del proyecto vital está mediado no sólo por las voluntades y las habilidades sino por el logro de oportunidades reales como respuesta a los procesos de desigualdad social (Criado, 2005; Medan, 2011). He ahí una manera de responder a estructuras sociales y económicas donde el acceso en condiciones de igualdad al trabajo o la educación, también debe partir desde las instituciones de las sociedades.

\subsection{ESTRUCTURAS DE TRANSICIÓN Y TIPOS DE TRAYECTORIAS VITALES}

Una vez se ha ampliado conceptualmente la idea de Proyecto de vida y sus dimensiones de construcción, es pertinente identificar las diferentes tipologías que se han desarrollado en torno a su realización. Se trata del concepto de estructuras de transición y trayectorias sociales. La primera se puede considerar como movimiento y la segunda se puede concebir como un proceso (Machado Pais, 2000). Una identifica las diferentes etapas de la vida donde se producen cambios significativos. Otra representa los caminos recorridos por el individuo en su curso vital en medio de determinaciones sociales y culturales. Es en la interacción de estas dinámicas que adquiere sentido una historia de vida protagonizada por un actor individual que tiene determinaciones estructurales pero que es capaz de actuar (Ghiardo y Dávila, 2008). Ahí es donde se configuran logros, metas y frustraciones del devenir personal.

Un aspecto hace referencia a la estructuras de transición que representa el cambio de condiciones en las distintas etapas de la vida, teniendo en cuenta los tiempos cronológicos y las estructuras sociales del contexto al que pertenece el individuo (Dávila y Ghiardo, 2012). En este momento cabe considerar por ejemplo el paso del estudio al fin del proceso formativo, del empleo al desempleo, de la dependencia a la independencia o de la no maternidad a la maternidad. En este sentido, esta articulación de procesos sociales e históricos articula las formas de transición en distintas etapas de la vida (Davila y Ghiardo, 2012). Así, se determinan las distintas fases en las que se desarrolla la vida humana y cada cambio de estado adquiere sentido por sí sólo.

Otro aspecto se vincula con las trayectorias sociales. A diferencia de las estructuras de transición donde cada etapa de la vida adquiere un sentido en sí mismo, desde el punto de vista de la trayectoria estos cambios adquieren interés a partir de valoraciones simbólicas y sociales. Así, estos cambios no se producen secuencialmente sino que se configuran a partir del grupo social de origen, el nivel de educación, el tipo de institución educativa, el título que otorga, el valor simbólico del título y el trabajo al que se accede con éste (Dávila, Guiardo y Medrano, 2005, Dávila y Ghiardo 2012). Es un proceso complejo que se produce en el marco de las tensiones entre las aspiraciones y la situación social del individuo. En este caso la trayectoria más que una historia vital es el reflejo de las estructuras sociales a las que pertenece (Machado Pais, 2000). Es de ese modo que se puede comprender el impacto del entorno colectivo en la orientación de los rumbos individuales. En este caso, 
la configuración exitosa o fallida de las trayectorias vitales, también dependerá de la herencia, acumulación, apropiación y transferencia de capitales económico, cultural, social y simbólico (Bourdieu, 1998, 2002; Bourdieu y Passeron, 1979, 2003). Su menor o mayor disponibilidad promoverá diversas oportunidades de movilidad social y de inclusión desde el acceso al trabajo o a la educación.

Desde esta perspectiva, se evidencian trayectorias de tipo lineal, reversible o tipo yo-yo. Las trayectorias lineales son las de final conocido, culturalmente aceptadas y socialmente reproducidas donde se produce la transición educación-empleo-matrimonio -niños (Du Bois-Reymond y López, 2004; Dávila y Ghiardo, 2005; Machado Pais, 2000; Sepúlveda, 2010). No obstante, teniendo en cuenta los condicionamientos económicos, la situación social y las dificultades para acceder al sistema educativo o laboral, se puede considerar que las trayectorias lineales no corresponden con la mayoría de las circunstancias. Se puede dar casos en que algunas personas logren sus objetivos vitales en medio de estos obstáculos. No obstante, esta excepción no hace más que confirmar las tensiones que suponen configurar sincrónicamente la dimensión personal, la profesional y la laboral. Es entonces cuando es pertinente hablar de trayectorias reversibles, laberínticas o tipo yo-yo donde la construcción del proyecto de vida implica mayor incertidumbre e imprevisibilidad (Machado Pais, 2000; Du Bois-Reymond y López, 2004). A veces el título no garantiza el acceso al mercado laboral, la vida laboral se puede compaginar con la académica o de una situación de ocupación laboral se puede pasar nuevamente al desempleo.

No obstante, más allá de la diferencia semántica, los dos procesos son complementarios entre sí y adquieren sentido cuando se relacionan (Davila y Ghiardo, 2012). De esta manera, la construcción de una historia vital, el cambio en cada etapa de la vida y las condiciones de acceder a ella, se produce a partir de la relación entre la acción consciente y la realidad presente. Por ello, la estructura de las transiciones puede tener algún impacto en las trayectorias dependiendo del grupo social de origen y éstas a su vez marcan las estructuras de transición (Davila et al., 2005). Por ejemplo, ser madre a temprana edad puede tener o no efecto en la trayectoria social de una joven si tiene los recursos económicos o el apoyo familiar para continuar su formación académica y poder acceder a mejores opciones de empleo.

\subsection{CARACTERÍSTICAS DEL PROYECTO DE VIDA}

El proyecto de vida comprende aspectos internos y externos de la vida humana (LomelíParga et al., 2016). Se relacionan con factores personales y sociales que determinan el curso de los itinerarios vitales. En este sentido, incluye el contexto, el plan vida, el sentido de vida, las capacidades, los objetivos, la autorreflexión, la autodeterminación personal, la autovaloración y la búsqueda de la autorrealización (D’Angelo, 2000, 2003). Con respecto a esto, se logra evidenciar la importancia de la construcción de sí mismo a partir de la reflexión sobre las fortalezas y los aspectos por mejorar con el fin de aprovechar las potencialidades y superar las dificultades. Además de los factores mencionados, se puede incluir el positivismo, el autoconocimiento, la autoestima, la visión y el compromiso (Vargas, 2005). Se confirma nuevamente la relación de la autorrealización a partir de un compromiso real con la idea de una proyección vital futura. En este sentido, estos factores implican una interacción con los valores personales (Castañeda, 2001). Así, las características de la personalidad entran en relación con las habilidades que se desarrollan a partir de las experiencias profesionales y personales. Por ello, la elaboración de un 
curso de vida no se puede desligar de la capacidad para elaborar y resolver conflictos adecuadamente (D’Angelo, 2003). Desde esta perspectiva, las características del proyecto de vida se pueden resumir a través de las siguientes:

\subsubsection{Valoración de sí}

En esta investigación la construcción de sí mismo se puede entender como la capacidad para comprender y valorar las capacidades individuales, con el fin de potencializar fortalezas y superar dificultades. Es posible englobar el concepto a través de la Autoestima y el Autoconocimiento. Desde la relación complementaria entre ambos aspectos el individuo se encamina hacia la realización personal.

En este caso la autoestima sería la actitud que confiere seguridad sobre la propia personalidad (Vargas, 2005). Es decir, el concepto implica también la idea del autocuidado como alternativa para el crecimiento personal. Esa sería una de las estrategias para conocerse, superar las dificultades y convertir las oportunidades en éxitos (Castañeda, 2001). Eso quiere decir que desde el autorreconocimiento, también se produce una sensibilización acerca de las propias realidades y posibilidades. De este modo, al ser consciente de sus propia cualidades, la persona se proyecta hacia el futuro, se impone a sí misma objetivos y actúa en favor de ellos al sentirse en capacidad para alcanzarlos (Alcántara, 2005). Por ello, la valoración de sí se constituye como uno de los elementos centrales en la construcción de ese proyecto de vida.

De igual forma, el autoconocimiento se constituye como posibilidad para conocer las posibilidades y transformar las debilidades en oportunidades de mejora (D'Angelo, 1986; D’Angelo, 2000). Es decir, éste actúa transformando la visión acerca del futuro que se puede construir al ayudar a descubrir potencialidades personales. Desde este enfoque, el conocimiento de sí ayuda a iniciar la planeación, tomar decisiones y proyectar la vida hacia la realidad que se quiere construir (Romero, 2004; Castañeda, 2001; Leiva, 2012). De esta forma, la autoestima y el conocimiento actúan como estrategia para construir el proyecto de vida de acuerdo con una perspectiva integral. Es por ello que un individuo se encamina hacia la autorrealización cuando es capaz de planear un plan vital de acuerdo con sus objetivos, expectativas y posibilidades reales (D'Angelo, 2000; Leiva, 2012). En efecto, desde la búsqueda de un sentido de vida al día a día también se puede estructurar la planeación del futuro.

\subsubsection{Proyección futura}

Es la relación que establece el individuo con el futuro desde el presente a través de las estrategias que utiliza y las habilidades que desarrolla para construir su plan vital. Se puede resumir a través de Visión, Objetivos, Estrategias y Habilidades. Desde la articulación de los cuatro factores se puede producir una acción concreta para la persecución de esa realidad a la que se aspira.

En este sentido, la visión articula el presente con las proyecciones futuras, motivando a la persona y convirtiendo el propósito en acción (Vargas, 2005). Por tanto, las acciones están orientadas hacia metas vitales de esa planeación personal, donde la expectativa se materializa desde las actividades llevadas a cabo para cumplir un ideal. Así, estos objetivos se constituyen como pautas de las estrategias a desarrollar en la construcción del itinerario 
vital (D’Angelo, 1986; Castro y Díaz, 2002; Vargas, 2005). No es éste en sí mismo sino la relación que establece con aspiraciones personales vitales. Así, requiere ser mensurable, realista y significativo para la persona con el fin de que adquiera valor motivacional para ella (Vargas, 2005). Es ahí donde el proyecto de vida adquiere un sentido vital y justifica su razón de ser como plan de acción personal orientado a determinar el curso de vida.

En esa medida, desde la interacción entre la visión, los objetivos y las estrategias, el plan de vida promueve una articulación desde la acción presente con el futuro al que se pretende llegar. Esto deriva en compromiso genuino, realista y responsable con el proceso de realización personal (Vargas, 2005; Castañeda, 2001). En conclusión, la proyección futura se construye desde la realidad presente como referente de las metas a las que se aspira y como punto de partida de las estrategias que se pretenden poner en práctica.

\subsubsection{Contexto social y relación con el entorno}

Define el contexto del individuo y sus condiciones socioeconómicas de existencia, determinando las posibilidades de acceder a procesos laborales o educativos y garantizando la inclusión social. Se puede resumir en Contexto y Recursos Económicos. De esta forma, el contexto influye en el proyecto de vida en la medida en que determinan el sentido en que crecen y se desarrollan las personas, estructurando la realidad de cada quien (Leiva, 2012). Teniendo en cuenta lo señalado, al hablar del entorno en que se desarrollan las historias vitales, es preciso tener en cuenta la influencia de las relaciones familiares e interpersonales.

Este entorno social está representado por diversos ámbitos de la vida humana. En efecto, la familia, las condiciones económicas, los amigos o los referentes culturales pueden tener un peso en la toma de decisiones o en los logros de las dimensiones laborales, profesionales o sociales (Casal et al., 2006; Ruiz, 2011). En este aspecto, la familia es un factor de partida y de permanencia constante en las orientaciones que puedan tomar los caminos individuales. Así es como el direccionamiento del futuro y las elecciones del presente también adquieren sentido desde las tensiones de la cotidianidad del hogar. Así, factores como el clima familiar, las rupturas familiares, el cambio de las relaciones entre los hermanos o padres y la variación de las condiciones económicas puede impactar en las alternativas o acciones que orienten la proyección vital (Casal et al., 2006). A la luz de estas dinámicas se puede comprender por ejemplo la inserción laboral a temprana a edad para colaborar con el sustento del hogar. La mayor duración en los años de estudio debido al poco apoyo familiar. $\mathrm{O}$ también el abandono y el fracaso escolar debido a la falta de acompañamiento familiar.

Desde esta mirada la acción individual también debe comprenderse en el marco de las oportunidades o los obstáculos que sugieren las realidades sociales. No es negar el potencial transformador sobre la propia realidad sino reconocer los condicionamientos concretos que pueden tener impacto en el curso vital. Es por esa razón que el contexto puede definir las posibilidades o limitaciones que tiene el individuo y determinar las direcciones esenciales del proyecto vital (D’Angelo, 2003). Desde la contradicción entre la proyección las acciones, las circunstancias reales de existencia y los ideales, el individuo va construyendo su futuro. De esa manera, se evidencia una permanente comunicación individuo - sociedad en el desarrollo del itinerario de vida (D’Angelo, 2003). Es en esas intermediaciones entre lo real y lo posible o entre el obstáculo y la expectativa donde el individuo se construye a sî mismo de una manera constante, compleja y en tensión con las realidades de su contexto. 


\section{METODOLOGÍA}

Para el desarrollo de la investigación, se recurrió a la metodología del estudio de casos como modelo de aproximación social, significativa y científica al tema objeto de estudio. De acuerdo con Yin, el estudio de casos es una técnica de investigación apropiada porque permite indagar sobre un fenómeno contemporáneo en su contexto real, utilizando múltiples fuentes de datos, así como uno o varios casos de estudio (1989). En esto coincide Einsenhard quien entiende el estudio de casos como una estrategia de investigación adecuada para comprender sustancialmente las dinámicas particulares a contextos específicos, contrastando casos y fuentes de información cualitativas y cuantitativas para generar respuestas, responder preguntas o contrastar datos (1989). Acá el estudio se centra en la Institución Educativa el Playón y su comunidad educativa con el objetivo de realizar un análisis en profundidad en torno al problema de investigación.

En este sentido, para investigar el papel de la escuela en la construcción de los proyectos de vida, el estudio de caso es una técnica pertinente en tanto que permite conocer a fondo sus particularidades así como la obtención de datos cuantitativos para el análisis. Además, debido a la diversidad de los datos en que se basa el estudio y al tener en cuenta diversas perspectivas, es posible comprender cómo y porqué ocurren los fenómenos estudiados (Chetty, 1996). Ello es particularmente necesario en este estudio, donde se pretende comprender el papel de la escuela y de su comunidad educativa en los cursos vitales de sus estudiantes. Así, el rigor científico del estudio de caso en esta investigación, se valora por ser contrastable con datos cualitativos y cuantitativos obtenidos a partir de un análisis sustancial desde su contexto particular así como por su posibilidad de transferencia a otros escenarios.

De esta manera, al realizar este estudio, se utilizan cuatro técnicas para la obtención de información: observación participante, entrevistas (en profundidad y estructuradas), grupos de discusión y análisis de documentación. Por tanto, se espera que esta combinación contribuya a realizar un proceso de reflexión riguroso. Con respecto a este punto, la triangulación de datos permite obtener una comprensión más clara del escenario estudiado (Taylor y Bogdan, 1987). Es una manera de tener en cuenta todas las posibles variables que respondan al problema de investigación, contrastar las ideas construidas previamente y resolver los objetivos planteados.

\subsection{OBJETO DE ESTUDIO}

El tema objeto de estudio de la investigación se centra en el análisis de las dinámicas de interacción social en las escuelas del Municipio de Carepa, Región de Urabá, Departamento de Antioquia (Colombia). En concreto, se trata de uno de los lugares que más sufrió las repercusiones del conflicto armado interno. Para ello, se toma la idea del "conflicto", como referente de indagación en torno al papel de la escuela frente a la reproducción de las problemáticas de una comunidad o a la transmisión de valores y pautas de comportamiento que validan estas tensiones. Desde esta perspectiva, se pretende indagar en qué medida las interacciones sociales en las escuelas del Municipio de Carepa, responden a dos procesos complementarios entre sí. En primer lugar, ante la escuela como escenario catalizador de conflictos y representación a escala del referente de valores y comportamientos de una comunidad. En segundo lugar, ante la escuela como posible escenario de empoderamiento y construcción del proyecto de vida. 


\subsection{POBLACIÓN}

Esta investigación se realizó con 20 estudiantes de la Institución Educativa El Playón, Municipio de Carepa, Región de Urabá, Departamento de Antioquia, Colombia. La población está compuesta por 12 mujeres y 8 hombres. Con respecto a sus edades, hay un estudiante de 10 años, uno de 11 años, 2 de 12 años, 3 de 13 años, 1 de 14 años 4 de 15 años, 3 de 17 años y 5 de 18 años. En cuanto al nivel de escolaridad, los estudiantes pertenecen a la Secundaria. De esta manera, 2 estudiantes pertenecen al grado $6^{\circ}, 6$ al grado $7^{\circ}, 2$ al grado $8^{\circ}, 2$ al grado $10^{\circ}$ y 8 al grado $11^{\circ}$. Por último, participaron 8 docentes, 5 hombres y 3 mujeres. Entre los hombres, se encuentra el Rector y el Coordinador Académico.

\subsection{FASES DE LA INVESTIGACIÓN}

Esta investigación se desarrolló a partir de dos fases. La primera tuvo lugar entre enero de 2013 y noviembre de 2014. Como técnicas para la recogida de datos se tuvo en cuenta la observación etnográfica y la observación participante a partir de una convivencia con la comunidad. La segunda se realizó entre diciembre de 2014 y diciembre de 2016 y corresponde con el cambio de residencia desde la región de Urabá hacia la ciudad de Medellín. Esta fase de la investigación, se desarrolló a partir de varios viajes a la región para hacer las entrevistas, los grupos de discusión y la observación participante.

\subsection{PROCEDIMIENTOS METODOLÓGICOS}

Para definir las tensiones en la construcción del Proyecto de vida, se recurrió a dos fases. En primer lugar, se utilizó la entrevista estructurada para obtener una serie de datos cuantificables, verificables y facilitar su sistematización. Para esto se construyó un Instrumento de Análisis del Proyecto de Vida. Este instrumento incluye dos momentos. Primero, una parte valorativa de la construcción del proyecto de vida que incluye preguntas sobre tres aspectos: Valoración de sí mismo, Proyección futura y Contexto social y relación con el entorno. Estos aspectos fueron retomados a partir de la conceptualización teórica que se hizo en el apartado anterior. El segundo momento del Instrumento incluye una escala de Likert donde se analizan las ocho características que implican la construcción del Proyecto de Vida de acuerdo con la conceptualización teórica anterior. Así, se incluyen las siguientes:

- Autoestima, Autoconocimiento, Visión, Objetivos, Estrategias, Entorno, Habilidades, Contexto, Recursos económicos.

En segundo lugar, se hicieron cuatro grupos de discusión para obtener datos cualitativos y soportar la interpretación de la información cuantitativa. Además, se hicieron las entrevistas en profundidad con los docentes. Es decir, desde el uso de una metodología mixta se pretende desarrollar un proceso de análisis riguroso, científicamente objetivo pero pertinente al contexto social de donde surge la información y al problema de investigación (Heinz, 2003). 


\subsection{SISTEMATIZACIÓN Y ANÁLISIS DE LOS DATOS}

Para sistematizar la información, primero se procedió a hacer la sumatoria de los resultados de cada estudiante por cada una de las características del proyecto de vida a partir de los datos de la escala de Likert. Esto se hizo para obtener un resultado global e inicial sobre los principales aspectos que los estudiantes consideran determinantes en la construcción del proyecto de vida. De esta se encontró que la visión, las metas y las estrategias adquieren interés central por parte de los estudiantes. Por ello, los datos que arrojaron esta fase se utilizaron para estructurar las preguntas de los grupos de discusión.

Tabla 1: Características del Proyecto de Vida

\begin{tabular}{|c|c|c|c|c|c|c|c|c|c|}
\hline \multicolumn{10}{|c|}{ CARACTERISTICAS DEL PROYECTO DE VIDA } \\
\hline Estudiante $\mathrm{N}^{\circ}$ & 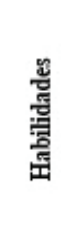 & 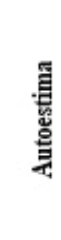 & 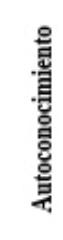 & 淃 & 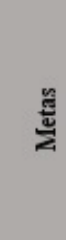 & 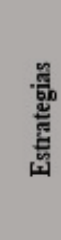 & 월 & 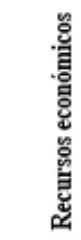 & 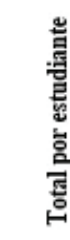 \\
\hline 1 & 7 & 7 & 7 & 7 & 8 & 8 & 9 & 7 & 60 \\
\hline 2 & 7 & 5 & 5 & 8 & 8 & 8 & 8 & 8 & 57 \\
\hline 3 & 8 & 7 & 7 & 10 & 10 & 10 & 8 & 8 & 68 \\
\hline 4 & 8 & 7 & 7 & 8 & 8 & 9 & 7 & 7 & 61 \\
\hline 5 & 7 & 7 & 7 & 8 & 8 & 8 & 8 & 7 & 60 \\
\hline 6 & 7 & 6 & 7 & 7 & 8 & 8 & 7 & 8 & 58 \\
\hline 7 & 8 & 8 & 8 & 6 & 6 & 6 & 7 & 7 & 56 \\
\hline 8 & 6 & 6 & 7 & 7 & 7 & 6 & 7 & 8 & 54 \\
\hline 9 & 10 & 9 & 9 & 9 & 10 & 10 & 8 & 7 & 72 \\
\hline 10 & 6 & 7 & 7 & 7 & 7 & 7 & 7 & 8 & 56 \\
\hline 11 & 7 & 7 & 7 & 8 & 8 & 7 & 7 & 7 & 58 \\
\hline 12 & 8 & 8 & 7 & 8 & 8 & 7 & 7 & 7 & 60 \\
\hline 13 & 8 & 9 & 9 & 10 & 10 & 10 & 8 & 7 & 71 \\
\hline 14 & 9 & 9 & 9 & 5 & 5 & 5 & 7 & 7 & 56 \\
\hline 15 & 7 & 7 & 7 & 7 & 8 & 8 & 7 & 8 & 59 \\
\hline 16 & 5 & 5 & 6 & 7 & 7 & 7 & 7 & 7 & 51 \\
\hline 17 & 7 & 10 & 9 & 10 & 10 & 10 & 8 & 7 & 71 \\
\hline 18 & 7 & 7 & 6 & 6 & 7 & 7 & 8 & 8 & 56 \\
\hline 19 & 7 & 7 & 7 & 8 & 8 & 8 & 9 & 8 & 62 \\
\hline 20 & 7 & 7 & 7 & 7 & 8 & 7 & 7 & 7 & 57 \\
\hline $\begin{array}{c}\text { Total por } \\
\text { característica del } \\
\text { proyecto de vida }\end{array}$ & 146 & 145 & 145 & 153 & 159 & 156 & 151 & 148 & \\
\hline
\end{tabular}

Fuente: elaboración propia 
Después se procedió a sistematizar los datos recogidos en la entrevista estructurada. Así, se realizó la transcripción de los cuatro grupos de discusión para facilitar la clasificación y el análisis de la información. Posteriormente, se hizo una lectura inicial de los datos para codificar la información. Esto permitió identificar los principales temas que surgieron a partir del proceso de recolección de información. Es decir, se procedió a la categorización de la información como proceso que posibilita identificar las principales categorías y subcategorías de análisis (Osses, Sánchez y Ibánez, 2006). Estos estaban referidos principalmente a aspectos que implican la construcción del proyecto de vida y su relación con el contexto social. Posteriormente, se procedió a codificar los datos al relacionar los datos recogidos con las categorías construidas previamente. En efecto, la codificación es un proceso que permite la reunión y el análisis de datos que se refieren a un tema, idea o concepto común (Taylor y Bogdan, 1987; Álvarez-Gayou, 2003; Fernández, 2006). De esta manera, se construyó una Matriz de análisis que arrojó las siguientes categorías y subcategorías:

Tabla 2: Matriz de análisis

\begin{tabular}{|c|c|c|}
\hline \multicolumn{3}{|c|}{ Matriz de análisis } \\
\hline Categorías & Subcategorías & Análisis de la categoría y las subcategorías \\
\hline \multirow[b]{2}{*}{$\begin{array}{l}\text { Valoración de } \\
\text { sí mismo }\end{array}$} & Autoestima & \multirow{2}{*}{$\begin{array}{l}\text { Según los estudiantes, el fortalecimiento de la autoesti- } \\
\text { ma y el autoconocimiento personal, se convierten en } \\
\text { dos retos para incidir en la construcción del Proyecto de } \\
\text { Vida. }\end{array}$} \\
\hline & Autoconocimiento & \\
\hline \multirow{3}{*}{$\begin{array}{l}\text { Proyección } \\
\text { futura }\end{array}$} & Visión & \multirow{3}{*}{$\begin{array}{l}\text { De acuerdo con los estudiantes, la visión y los objetivos } \\
\text { vitales deben ser coherentes con estrategias concretas } \\
\text { en favor del Proyecto de Vida que se aspira construir. }\end{array}$} \\
\hline & Objetivos & \\
\hline & Estrategias & \\
\hline \multirow{4}{*}{$\begin{array}{l}\text { Contexto } \\
\text { social y } \\
\text { sistema } \\
\text { educativo }\end{array}$} & Recursos económicos & \multirow{2}{*}{$\begin{array}{l}\text { Los estudiantes plantean que la construcción de su Pro- } \\
\text { yecto de Vida está condicionada por las limitaciones } \\
\text { sociales y económicas propias del contexto vulnerable } \\
\text { en que se desenvuelven. }\end{array}$} \\
\hline & Contexto & \\
\hline & $\begin{array}{l}\text { Proyecto de Vida } \\
\text { Alternativo (PVA) }\end{array}$ & $\begin{array}{l}\text { De acuerdo con estos planteamientos, se evidencia un } \\
\text { proceso alterno donde se produce la construcción de } \\
\text { "Proyectos de Vida Alternativos" en los que la niñez y } \\
\text { la adolescencia, están condicionadas por las circunstan- } \\
\text { cias sociales y se alternan simultáneamente con las res- } \\
\text { ponsabilidades de la vida adulta. }\end{array}$ \\
\hline & Sistema educativo & $\begin{array}{l}\text { A partir de las entrevistas a docentes y estudiantes, se } \\
\text { evidencia que el papel de la escuela en estos contextos } \\
\text { es reproducir estas dificultades sociales estructurales o } \\
\text { garantizar una educación integral y diferenciadora que } \\
\text { aporte significativamente al curso vital del joven. }\end{array}$ \\
\hline
\end{tabular}

Fuente: elaboración propia 


\section{ANÁLISIS Y RESULTADOS}

Al hacer una primera observación sobre los resultados que otorgan los estudiantes a los diferentes factores que se pueden tener en cuenta al momento de pensar en la construcción del proyecto de vida, se evidencia una relación a partir de tres dimensiones. Es decir, se evidencia una relación a partir de tres dimensiones: la valoración de sí mismo, la proyección futura y el contexto social y la relación con el entorno. Es posible pensar que en el marco de esa interacción se produce la construcción de un proyecto de vida que en el marco de un contexto vulnerable como el que se analiza, se comprende a partir de la tensión entre las metas vitales y el entorno social en el que se construye ese itinerario vital. En este sentido, la proyección vital es algo complejo, contradictorio, sujeto a las dinámicas económicas del entorno y a las problemáticas de las estructuras sociales heredadas de un conflicto que se ha desarrollado durante 40 años en el Urabá Antioqueño. Esta perspectiva se puede identificar a través de la siguiente representación gráfica:

Gráfica 1: Características del Proyecto de Vida

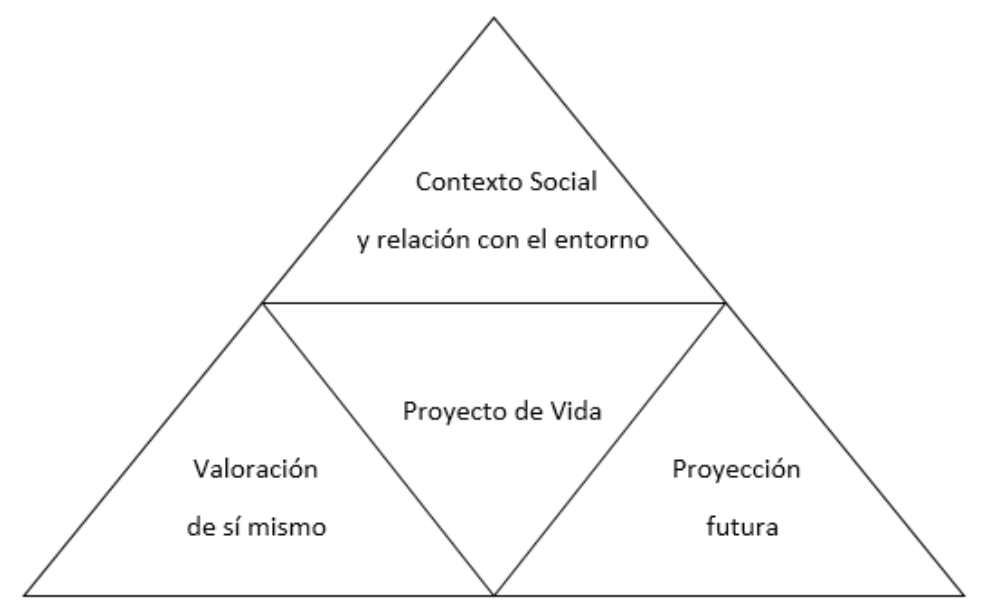

Fuente: elaboración propia

Esto hace pensar que el Proyecto de Vida en el Urabá Antioqueño no es algo unidireccional, seguro y certero, sino que está sujeto a las dinámicas económicas y sociales de la región. Más allá de representar una trayectoria lineal de la construcción del proyecto de vida, implica una alternancia de las estrategias para conseguir los objetivos vitales con los recursos económicos de los que dispone la familia y de las oportunidades o limitaciones que determine el entorno inmediato. A continuación se analizan los datos obtenidos en las entrevistas. 


\subsection{LA VALORACIÓN DE SÍ MISMO: CONSTRUCCIÓN DEL PROYECTO VITAL Y POSIBILIDADES DE AUTORREALIZACIÓN EN UN CONTEXTO VULNERABLE}

Al momento de pensar en la construcción del proyecto de vida de los jóvenes con los que se ha realizado esta investigación, es pertinente preguntarse acerca del papel de la autoestima y el autoconocimiento en el planteamiento iniciar de un itinerario vital. Si bien las posibilidades de realización o no de esa proyección, depende de las oportunidades reales que ofrezca el entorno, para la mayoría de estos jóvenes adquiere una importancia central la forma de aprender a valorarse y a reconocerse desde sus aspiraciones personales. Es precisamente desde el reconocimiento de lo que se posee, de lo que no, y de lo que se pretende llegar a ser, la forma a través de la cual se puede comenzar a formar una planeación futura sobre el curso vital. Esta posibilidad de reconocerse se denomina en esta investigación como valoración de sí mismo. Este concepto se puede entender como proceso a través del cual el ser humano reconoce sus debilidades para mejorarlas y sus habilidades para potenciarlas con el fin de identificar esas estrategias de autorrealización personal.

\subsection{PROYECCIÓN FUTURA: PAPEL DE LOS OBJETIVOS Y LAS ESTRATEGIAS VITALES FRENTE AL ROL DEL ENTORNO SOCIAL}

La proyección futura se comprende en esta investigación como la interacción entre los objetivos, la visión, las estrategias y las habilidades en el marco de la construcción de un proyecto de vida, teniendo en cuenta los condicionamientos sociales y económicos que pueden impactar en el rumbo que tome la construcción de este itinerario vital. No obstante, más allá de funcionar como guía orientadora de acciones, cuando se trata de estos jóvenes del Urabá Antioqueño, para la mayoría la construcción de una visión resulta un proceso complejo.

Este planteamiento se explica por dos circunstancias. Por un lado, el presente se plantea como momento a partir del cual se planea la construcción de un itinerario vital. Es decir, más allá de actuar desde el futuro al que se aspira llegar, estos jóvenes construyen su curso vital a partir del día a día y de la relación con su entorno próximo. En esa medida, la posibilidad de tener buenas relaciones en el entorno familiar o social, así como la oportunidad de obtener un buen desempeño académico, se convierten en factores que median en ese proyecto. Proyección vital que en este caso es pensada por estos jóvenes desde el corto plazo. Por otro lado, la juventud se manifiesta precisamente como momento de la vida en el que apenas se están definiendo intereses, expectativas y preferencias. Concretamente, apenas se están formando las características que identifican una personalidad y por eso se hace complicado para ellos pensar a partir del futuro al que se pretende llegar. En ambos casos el presente se convierte en factor que guía las acciones que cimientan el curso de vida.

De igual manera, el contexto social se convierte en factor que hace compleja la posibilidad de pensar en el futuro en términos de certeza. Las dificultades económicas y la desestructuración familiar, hacen inevitable el planteamiento de un proyecto de vida a partir de la incertidumbre. De ningún modo se niega la posibilidad de tener aspiraciones de vida, pero el factor económico y familiar siempre aparece presente como condicionamientos reales frente a esos ideales a los que se aspira.

Es posible concluir que la proyección futura se puede comprender a partir del conjunto de estrategias desplegadas para la consecución de los objetivos vitales en el marco de la 
contradicción entre las aspiraciones personales y los condicionamientos del entorno social. Entre los jóvenes también es un proceso mediado por una visión de vida e identidad todavía en proceso de construcción. Además, en el caso del Urabá Antioqueño, la posibilidad de aspirar a esas metas vitales también está condicionada por factores sociales y económicos, según se analiza a continuación.

\subsection{RECURSOS ECONÓMICOS, CONTEXTO SOCIAL Y SISTEMA EDUCATIVO}

No obstante, al pensar en la construcción del proyecto de vida, es necesario tener en cuenta que más allá de la proyección futura, las posibilidades de autorrealización también van a depender de las oportunidades o limitaciones que ofrezca el contexto al cual pertenece el individuo. Es decir, las aspiraciones y realizaciones personales se van constituyendo a partir de las relaciones que el individuo establece con el territorio o desde las realidades que ofrece su entorno. En contextos vulnerables estas interacciones suelen ser complejas y limitadas por dinámicas económicas y sociales.

De esta manera, en el itinerario vital se evidencia una interacción permanente entre dos aspectos. En primer lugar, por factores estructurales como los recursos económicos y el acceso a medios de apoyo financiero. En segundo lugar, por factores subjetivos que pueden hacer variar las condiciones económicas o sociales, en función de las relaciones establecidas con el contexto social, familiar o laboral. Ambas dinámicas definen la construcción de proyectos vitales no lineales que se van configurando a partir de una constante tensión entre las estrategias por obtener los objetivos vitales y las posibilidades concretas de realización en el marco de unos condicionamientos concretos.

En un contexto social complejo como el analizado, el sistema educativo también juega un papel esencial. De su mano, se encuentra la posibilidad de condicionar o favorecer la construcción del proyecto de vida de los jóvenes. De acuerdo con los docentes entrevistados, hay tres factores que limitan el papel de la institución educativa en este proceso. En primer lugar, se valora el papel del docente que se impone ante los directivos, los padres de familia y los estudiantes a través de la figura de poder que impone miedo más que respeto. En segundo lugar, se requiere mayor cualificación en los docentes pues en la región, algunos de ellos sólo han terminado el Bachillerato o son Normalistas. Por último, hace falta promover un proceso formativo integral que favorezca equitativamente el aprendizaje y el desarrollo de habilidades sociales.

No obstante, hay casos en donde el sistema educativo impacta positivamente en algunos estudiantes. De acuerdo con los docentes entrevistados, esto se explica particularmente por tres factores. En primer lugar, el profesor asume la figura de líder que motiva procesos orientados a fortalecer saberes y habilidades de los estudiantes. En segundo lugar, está bien cualificado en cuanto a su área del saber y sus técnicas pedagógicas y didácticas. Por último, favorece procesos educativos integrales que incluyen el aprendizaje de conocimientos y el desarrollo de habilidades sociales. Esto garantiza un beneficio positivo en la construcción de algunos proyectos de vida, pues se fomenta el desarrollo de capacidades para poner en práctica a nivel familiar, laboral y social. 


\section{EL PROYECTO DE VIDA ALTERNATIVO (PVA)}

En el Urabá Antioqueño, algunos de los factores determinantes en la construcción del proyecto de vida, son los condicionamientos estructurales impuestos por la posesión de recursos económicos, las fuentes de empleo familiar o personal, el contexto social y el sistema educativo. De esta manera, más allá de las expectativas frente al curso vital, existe una consciencia real acerca de los posibles obstáculos en la búsqueda de la autorrealización personal. Al sistematizar los factores que influyen en la trayectoria vital de los jóvenes del Urabá, se evidencian tres componentes a partir de los que se produce la construcción alternativa del proyecto de vida. Se trata del entorno y las relaciones interpersonales, de las limitaciones económicas y las estrategias de trabajo informal, así como de la perspectiva de género. De manera gráfica, se podrían representar así:

Gráfica 2: Características del Proyecto de Vida Alternativo (PVA)

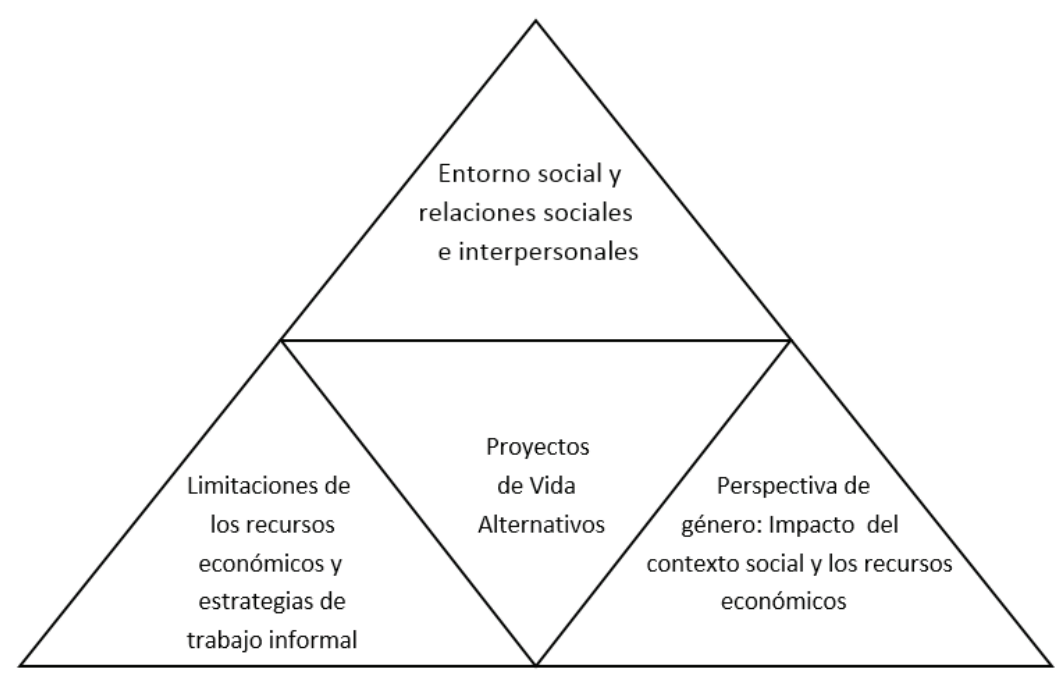

Fuente: Elaboración propia

\subsection{LIMITACIONES DE LOS RECURSOS ECONÓMICOS Y ESTRATEGIAS DE TRABAJO INFORMAL EN LA CONSTRUCCIÓN DEL PROYECTO DE VIDA}

Al pensar en las estrategias desplegadas por los estudiantes ante la escasez económica individual o familiar, se comprenden las dimensiones de lo que implica la construcción del proyecto de vida en el Urabá Antioqueño. En este sentido se identifican unas coyunturas vitales donde las etapas tradicionales de la infancia y la adolescencia, se alternan con las responsabilidades económicas y laborales de la vida adulta. Por tanto, para la mayor parte de los estudiantes que hacen parte de esta investigación, la mayor o menor disponibilidad de recursos económicos tiene un papel fundamental en el curso vital. Así es posible identificar que la mitad de ellos ya trabajan en actividades económicas informales. 
Por ello, al analizar el impacto de la construcción de un proyecto de vida donde la niñez y la juventud se alternan con las responsabilidades propias de adultez, es preciso resaltar que esto tiene repercusiones en dos ámbitos. En primer lugar, desde el ámbito escolar, esto tiende a complicar el desempeño de las actividades académicas pues estos jóvenes suelen tener menos tiempo para desarrollar sus tareas escolares. Así, estudian en la mañana, trabajan en la tarde y hacen sus deberes académicos en la noche, por lo cual al día siguiente llegan cansados a estudiar. De igual manera, algunas de estas actividades laborales a veces se realizan en horarios de estudio, por lo cual en ocasiones esto es causa de absentismo escolar. En segundo lugar, desde el ámbito personal, esta alternancia puede ocasionar repercusiones positivas pues si bien para algunos esto interfiere en el ámbito académico, para otros esto es una manera de desarrollar sus habilidades y capacidades. Efectivamente, al adquirir responsabilidades de la vida adulta, algunos de ellos desarrollan mayor compromiso, autoconfianza y empoderamiento al actuar desde la autonomía en la construcción de su proyecto de vida.

\subsection{ENTORNO SOCIAL Y RELACIONES SOCIALES E INTERPERSONALES}

En segundo lugar, también se evidencian factores de orden social y familiar que tienen un papel en la construcción del proyecto de vida. En el caso de los estudiantes con los cuales se ha realizado esta investigación, se trata de entornos familiares complejos y desarticulados. En la mayor parte de los casos, se trata de niños y jóvenes que viven principalmente con su madre y con su padrastro. Es pertinente destacar que dependiendo de la formación académica de la familia, también se recibe un mayor o menor acompañamiento familiar.

De igual forma, la familia y los amigos adquieren un papel en la construcción del proyecto de vida, si se tiene en cuenta la influencia que pueden tener en la toma de decisiones. En efecto, esto permite evidenciar nuevamente cómo al momento de desarrollar las acciones orientadas a obtener los objetivos vitales, también entra en juego el rol que tienen los familiares o los amigos en las elecciones personales. De esta manera, el familiar representa la zona de confort y de seguridad ante la que es posible obtener seguridad, confianza, apoyo y buen consejo frente a los hechos del día a día. De igual modo, se evidencia una percepción sobre lo que representaría el mal amigo. Según estas apreciaciones, sería aquél que no ofrece solidaridad, actúa desde la envidia y se convierte en una mala influencia al momento de actuar o tomar decisiones. En consecuencia, la familia, los buenos amigos y las personas positivas son aquellas que podrían ser tenidas en cuenta al momento de tomar decisiones vitales y seguir el curso de la vida.

Es así que se evidencia una interrelación con la familia y el entorno social como factores que juegan parte en la construcción del proyecto vital. El soporte familiar y las interacciones al momento de orientar decisiones son importantes si se tiene en cuenta su impacto en la determinación del rumbo que tomen las acciones personales. Por ello, la configuración que adquiera el curso vital no se puede entender en sí misma, sino a partir de su relacionamiento con las realidades familiares y sociales. Contexto que suele ser complejo, contradictorio y está sujeto a las dinámicas del diario vivir. En este caso, entran en juego las aspiraciones vitales, las estrategias concretas para su realización y las posibilidades que ofrezcan los condicionamientos específicos de una comunidad. Es en el marco de estas coyunturas donde se construyen estos proyectos de vida. Proyecciones vitales complejas, no lineales y vinculadas a unos procesos histórico-sociales. No es negar 
Estudios Pedagógicos XLIII, Nº 3: 153-173, 2017

CONSTRUCCIÓN DE PROYECTOS DE VIDA ALTERNATIVOS (PVA) EN URABÁ, COLOMBIA: PAPEL DEL SISTEMA EDUCATIVO EN CONTEXTOS VULNERABLES

la posibilidad autorrealizadora del individuo sino tener en cuenta cómo su existencia se configura a partir de estas relaciones.

\subsection{PERSPECTIVA DE GÉNERO: IMPACTO DEL CONTEXTO SOCIAL Y LOS RECURSOS ECONÓMICOS EN LA CONSTRUCCIÓN DEL PROYECTO DE VIDA}

Por último, la perspectiva de género es un factor que también toma parte en la construcción del proyecto de vida. En el Urabá Antioqueño, es uno de los factores que determina el proceso de transición desde la educación primaria hacia la educación secundaria o incluso desde la educación secundaria al mundo laboral. No obstante, se tiene en cuenta el planteamiento de que el curso vital no es una configuración lineal y está sujeta al entorno social del cual hace parte el individuo. En ese sentido, es preciso tener en cuenta que las trayectorias de las niñas y jóvenes en esta región están expuestas a coyunturas complejas y contradictorias que se relacionan entre sí. Por ello, no se da una transición lineal desde la infancia, pasando por las etapas de la educación básica, para transitar hacia el mundo laboral y la construcción de una familia, sino que estos momentos vitales se alternan simultáneamente con el estudio, el trabajo y la conformación de una familia. Acá es un factor determinante el hecho de ser madre a temprana edad o de ser madre soltera.

Como se puede ver, en contextos vulnerables, el factor género adquiere un impacto en los proyectos vitales. Así, éstos no son una construcción que depende de la edad y de las aspiraciones sociales, sino de las posibilidades económicas y de la escaza gama de oportunidades laborales para las mujeres de la región. Son proyectos de vida que entran en contradicción con la idea del curso vital como conjunto de estrategias y acciones en favor de los objetivos vitales. De una manera más realista, es un proceso a través del cual se configura el curso vital, teniendo en cuenta las oportunidades y posibilidades que ofrece el contexto social y económico. Así, las aspiraciones vitales y las estrategias concretas para obtenerlas, hacen desplegar un conjunto de actividades económicas que generan una interrupción en la transición normal desde la niñez hacia la juventud. En este caso, la niñez se solapa con responsabilidades propias de la vida adulta y laboral, haciendo desplegar un conjunto de compromisos que explican la actitud proactiva de estas estudiantes frente a las realidades de sus familias y su entorno escolar.

\section{CONCLUSIONES}

La tensión entre las aspiraciones vitales y las limitaciones o las posibilidades que ofrece el entorno social y económico, define la configuración del Proyecto de Vida en el Urabá Antioqueño. En este caso suele ser complejo, no es lineal y es transversal. Adquiere múltiples dimensiones donde la transición tradicional desde la niñez a la adolescencia, está distorsionada por la adquisición de responsabilidades de la vida adulta. Más allá de construir un curso vital donde la proyección futura y la valoración de sí mismo determinen el rumbo de la existencia, las posibilidades reales de autorrealización están condicionadas por los recursos económicos, el contexto social y la relación con el entorno.

De esta manera, una de las características de estas trayectorias vitales es que están sujetas a la incertidumbre. En efecto, estos cursos vitales adquieren sentido a partir de su relación con la fluctuación de los recursos económicos, el apoyo familiar y la búsqueda de 
estrategias de autosostenimiento. Desde esta perspectiva, la creatividad, la iniciativa y el rebusque $^{1}$, surgen como estrategia para atender a las necesidades del hogar y al costeo de los materiales escolares.

En efecto, en estos cursos vitales juega un papel esencial la escuela. Después del hogar y el entorno social próximo, corresponde a ella otorgar las herramientas para que el estudiante pueda construir un proyecto de vida desde la autonomía y la proactividad. En contraposición del sistema educativo que favorece el aprendizaje repetitivo y valora al docente autoritario, ésta debe garantizar que el alumno desarrolle integralmente saberes, habilidades y capacidades para poner en práctica en su cotidianidad. Ya sea desde las relaciones interpersonales o desde las estrategias de trabajo informal que el joven despliega para garantizar su propio sostenimiento y el de su familia.

Es ahí cuando surge la idea de Proyectos de Vida Alternativos (PVA). De acuerdo con esta investigación, un proyecto de vida alternativo no niega la posibilidad de poder actuar en favor de los objetivos vitales. Por el contrario, hace referencia a un proceso alterno donde la vida académica de la niñez y la adolescencia está condicionada por las circunstancias sociales y se alterna simultáneamente con las responsabilidades de la vida adulta a través de diversas estrategias de autosostenimiento. En este sentido, la búsqueda de las metas personales y la colaboración con el sustento del hogar se complementa con el proceso de formación en el ámbito escolar. Por ello, más allá de identificar las dimensiones de un Proyecto de Vida ideal y lejano, se logran establecer las dimensiones de un curso vital que se construye en el día a día.

De esta manera, es posible concluir que el papel del sistema educativo en contextos vulnerables consiste en impactar de manera significativo en la construcción de los Proyectos de Vida Alternativos. Para ello, debe incidir en el desarrollo de habilidades, capacidades y potencialidades que fortalecen el empoderamiento del estudiante en su dimensión individual. En la misma medida, debe impactar en su mentalidad como estrategia para fortalecer la autoestima y la motivación frente a la propia trayectoria vital. Estos procesos de potenciamiento a nivel personal, no son garantía de éxito laboral o económico en el futuro cercano. Sin embargo, impactan en el fortalecimiento de la autonomía al momento de tomar decisiones y de actuar de una manera crítica en favor de unos objetivos propuestos. De igual manera, posibilitan al estudiante hacer ejercicio de su liderazgo impactando en su entorno familiar y escolar. En este sentido, el papel de la escuela en contextos vulnerables consiste en reproducir las dificultades sociales estructurales de un contexto o garantizar una formación integral y diferenciadora que aporte significativamente al curso vital del joven.

\section{REFERENCIAS BIBLIOGRÁFICAS}

Alcántara, A. (2005). Educar la autoestima: Métodos, técnicas y actividades. Barcelona: CEAC. Álvarez-Gayou, J. (2003). Cómo hacer investigación cualitativa. Fundamentos y metodología. México D.F.: Paidós.

Bourdieu, P. (1998). Capital cultural, escuela y espacio social. México D.F.: Siglo XXI. Bourdieu, P. (2002). La distinción: Criterios y bases sociales del gusto. México D.F.: Taurus. Bourdieu, P. y Passeron, J. (1979). La reproducción: Elementos para una teoría del sistema de

Término que en Colombia hace referencia a la creación de estrategias de microemprendimiento y comercio a nivel personal o familiar para contribuir al sustento personal o familiar. 
Estudios Pedagógicos XLIII, Nº 3: 153-173, 2017

CONSTRUCCIÓN DE PROYECTOS DE VIDA ALTERNATIVOS (PVA) EN URABÁ, COLOMBIA: PAPEL DEL SISTEMA EDUCATIVO EN CONTEXTOS VULNERABLES

enseñanza. Barcelona: Laia.

Bourdieu, P. y Passeron, J. (2003). Los herederos: Los estudiantes y la cultura. Buenos Aires: Siglo XXI.

Casal, J., García, M., Merino Pareja, R. y Quesada, M. (2006). Aportaciones teóricas y metodológicas a la sociología de la juventud desde la perspectiva de la transición. Papers: Revista De Sociologia, vol.79, 21-48.

Castañeda, L. (2001). Un plan de vida para jóvenes: ¿qué harás con el resto de tu vida?. México D.F.: Ediciones Poder.

Castro, A. y Díaz, J. (2002). Objetivos de vida y satisfacción vital en adolescentes españoles y argentinos. Psicothema, vol.14 (1), 112-117.

Chetty, S. (1996). The case study method for research in small-and medium-sized firms. International Small Business Journal, vol.15 (1), 73-85.

Criado, E. (2005). La construcción de los problemas juveniles. Nómadas, vol.23, 86-93.

D'Angelo, O. (2003). Proyecto de vida y desarrollo integral humano. Recuperado el 3 de julio de 2016 desde: http://biblioteca.clacso.edu.ar/Cuba/cips/20150429033758/ 07D050.pdf

D'Angelo, O. (1986). La formación de los proyectos de vida del individuo: Una necesidad social. Revista Cubana De Psicología, vol.3 (2), 31-39.

D’Angelo, O. (2000). Proyecto de vida como categoria básica de interpretación de la identidad individual y social. Revista Cubana De Psicología, vol.17 (3), 270-275.

D’Angelo, O. (2002). El desarrollo profesional creador (DPC) como dimensión del proyecto de vida en el ámbito profesional. Revista Cubana De Psicología, vol.19 (2), 106-114.

Dávila, O. y Ghiardo, F. (2005). Trayectorias, transiciones y condiciones juveniles en Chile. Nueva Sociedad, vol. 200, 114-126.

Dávila, Ó. y Ghiardo, F. (2012). Transiciones a la vida adulta: Generaciones y cambio social en Chile. Ultima Década, vol. 37, 69-83.

Dávila, O., Ghiardo, F. y Medrano, C. (2005). Los desheredados. Trayectorias de vida y nuevas condiciones juveniles. Valparaíso: Ediciones CIDPA.

Du Bois-Reymond, M. y López, A. (2004). Transiciones tipo yo-yo y trayectorias fallidas: Hacia las políticas integradas de transición para los jóvenes europeos. Revista De Estudios De Juventud, vol. $65,11-29$.

Elder, G. (1998). The life course as developmental theory. Child Development, vol.69 (1), 1-12.

Fernández, L. (2006). ¿Cómo analizar datos cualitativos?. Recuperado el 10 de julio de 2016 desde: http://www.ub.edu/ice/recerca/fitxes/fitxa7-cast.htm

Ghiardo, F. y Dávila, O. (2008). Trayectorias sociales juveniles. ambivalencias y discursos sobre el trabajo. Santiago de Chile: Instituto Nacional de la Juventud.

Heinz, W. (2003). Combining methods in life-course research: A mixed blessing. In W. Heinz, \& V. Marshall (Eds.), Social dynamics of the life course. Transitions, institutionalism and interrelations (pp. 73-90). New York: Aldine de Gruyter.

Johnson-Hanks, J. (2002). On the limits of life stages in ethnography: Toward a theory of vital conjunctures. American Anthropologist, vol.104 (3), 865-880.

Leiva, P. (2012). Cambios en los referentes para la construcción de proyectos de vida juveniles. Revista Tendencias y Retos, vol. 17(1), 93-103.

Lomelí-Parga, A., López-Padilla, M. y Valenzuela-González, J. (2016). Autoestima, motivación e inteligencia emocional: Tres factores influyentes en el diseño exitoso de un proyecto de vida de jóvenes estudiantes de educación media. Revista Electrónica Educare, vol.20 (2), 1-22.

Machado Pais, J. (2000). Las transiciones y culturas de la juventud: Formas y escenificaciones. Revista Internacional De Ciencias Sociales, vol.164, 89-101.

Machado Pais, J. (2002). Laberintos de vida: Paro juvenil y rutas de salida (jóvenes portugueses). Revista De Estudios De Juventud, vol.56 (2), 87-101.

Mayer, K. (2009). New directions in life course research. Annual Review of Sociology, vol.35, 413-433. 
Medan, M. (2012). ¿ Proyecto de vida? tensiones en un programa de prevención del delito juvenil. Revista Latinoamericana De Ciencias Sociales, Niñez y Juventud, vol.10 (1), 79-91.

Osses, S., Sánchez, I. y Ibáñez, F. (2006). Investigación cualitativa en educación: Hacia la generación de teoría a través del proceso analítico. Estudios Pedagógicos, vol.32 (1), 119-133.

Pardo, I. (1999). Jóvenes construyendo su proyecto de vida. Bogotá: Cooperativa Editorial Magisterio.

Romero, S. (2004). Aprender a construir proyectos profesionales y vitales. REOP-Revista Española De Orientación y Psicopedagogía, vol.15 (2), 337-354.

Ruiz, J. (2011). Proyecto de vida, relatos autobiográficos y toma de decisiones. Teoría y Praxis Investigativa, vol.6 (1), 27-34.

Santana, L., Feliciano, L. y Santana, A. (2012). Análisis del proyecto de vida del alumnado de educación secundaria. Revista Española De Orientación y Psicopedagogía, vol.23 (1), 26-38.

Santana, L. (2002). Mujeres, igualdad de oportunidades y transición sociolaboral. Revista De Educación, vol.327, 169-187.

Sepúlveda, L. (2010). Las trayectorias de vida y el análisis del curso de la vida como fuentes de conocimiento y orientación en las políticas sociales. Perspectivas, vol.21, 27-53.

Taylor, S. y Bogdan, R. (1987). Introducción a los métodos cualitativos de investigación. Barcelona: Paidós.

Vargas, R. (2005). Proyecto de vida y planteamiento estratégico personal. Recuperado el 12 de julio de 2016 desde: http://www.ntslibrary.com/Proyecto\%20de\%20Vida\%20y\% 20Planeamiento\%20 Estrategico\%20Personal.pdf

Yin, R. (1989). Case study research: Design and methods. Newbury Park: Sage publications. 
